\title{
Physical instrumental vetoes for gravitational-wave burst triggers
}

\author{
P. Ajith, ${ }^{*}$ M. Hewitson, J. R. Smith, H. Grote, and S. Hild \\ Max-Planck-Institut für Gravitationsphysik (Albert-Einstein-Institut) and Leibniz Universität Hannover, \\ Callinstrasse 38, 30167 Hannover, Germany
}

K. A. Strain

Department of Physics and Astronomy, University of Glasgow, Glasgow, G12 8QQ, United Kingdom and Max-Planck-Institut für Gravitationsphysik (Albert-Einstein-Institut) and Leibniz Universität Hannover, Callinstrasse 38, 30167 Hannover, Germany

(Received 8 May 2007; revised manuscript received 14 June 2007; published 17 August 2007)

\begin{abstract}
We present a robust strategy to veto certain classes of instrumental glitches that appear at the output of interferometric gravitational-wave detectors. This veto method is "physical" in the sense that, in order to veto a burst trigger, we make use of our knowledge of the coupling of different detector subsystems to the main detector output. The main idea behind this method is that the noise in an instrumental channel $X$ can be transferred to the detector output (channel $H$ ) using the transfer function from $X$ to $H$, provided the noise coupling is linear and the transfer function is unique. If a nonstationarity in channel $H$ is causally related to one in channel $X$, the two have to be consistent with the transfer function. We formulate two methods for testing the consistency between the burst triggers in channel $X$ and channel $H$. One method makes use of the null stream constructed from channel $H$ and the transferred channel $X$, and the second involves cross correlating the two. We demonstrate the efficiency of the veto by "injecting" instrumental glitches in the hardware of the GEO 600 detector. The veto safety is demonstrated by performing gravitational-wave-like hardware injections. We also show an example application of this method using 5 days of data from the fifth science run of GEO 600. The method is found to have very high veto efficiency with a very low accidental veto rate.
\end{abstract}

DOI: 10.1103/PhysRevD.76.042004

PACS numbers: 95.55.Ym, 04.80.Nn

\section{INTRODUCTION}

The existence of gravitational waves (GWs) is the last and most intriguing prediction of Einstein's general theory of relativity (GTR), which is yet to be verified by a direct observation. A worldwide network of GW detectors consisting of ground-based interferometers [1-4] and resonant bars [5-7], most of them operating at or close to their design sensitivity, has started looking for signatures of GWs produced by astrophysical and cosmological sources. Apart from providing excellent tests of GTR in the strongly gravitating regions [8], GW astronomy is expected to open a new window to the Universe providing unique information about various astrophysical phenomena. For example, GW observations from the final coalescence of neutron star binaries will shed light on the nuclear equation of state [9], and the GWs produced by a neutron star or small black hole spiraling into a massive black hole will provide a "map" of the spacetime geometry around the larger object [10]. Also, GW observations can be used to measure the Hubble constant, deceleration parameter, and cosmological constant [11-13]. Similarly, the observation of the stochastic GW background from the early Universe can test a number of current speculations about the very early Universe [14].

*Ajith.Parameswaran@aei.mpg.de
The best understood (and, perhaps, the most promising) astrophysical sources of GWs for ground-based interferometers are the inspiraling compact binaries consisting of black holes and/or neutron stars. What makes this class of sources extremely interesting is that the expected waveforms can be very accurately modeled (and easily parametrized by a few intrinsic parameters of the binary, like the component masses and spins) by approximation techniques in GTR. This allows the data analysts to use the matched filtering technique in order to extract the signal that is buried in the detector noise. Another important class of astrophysical sources which can be probed through the optimal filtering technique is spinning neutron stars and pulsars.

There are other classes of GW sources, like core collapse of massive stars in supernovae, binary black hole/ neutron star mergers, accretion induced collapse of white dwarf stars, Gamma ray bursts etc., for which the physics is largely unknown, or too complex to allow computation of detailed gravitational waveforms. These kinds of sources are generally classified as "unmodeled burst sources." GW observations from merging black hole binaries can potentially bring very useful insights to the nonlinear dynamics of the spacetime curvature as the two black holes convert themselves to form a single black hole. GWs from a Gamma ray burst are expected to carry detailed information about its source, which cannot be probed via electro- 
magnetic observations. Similarly, correlated neutrino and GW observations from a core-collapse supernova could bring interesting insights into the newborn neutron star/ black hole in the core of the supernova (see [15] for a detailed review).

Any possible GW signal coming from an astrophysical/ cosmological source is generally buried in the detector noise. To extract these GW signatures from the noise is a nontrivial data analysis problem. Indeed, if time evolution of the GW phase is accurately known, the optimal filter for searching for this signal buried in the noise is the wellknown matched filter. But, since matched filtering relies on the prior knowledge of the signal, it may not be the best detection strategy in the search for unmodeled, short-lived GW bursts. In the next section, we give a brief introduction to the data analysis for "unmodeled burst sources."

\section{The search for transient, unmodeled gravitational-wave bursts}

One class of search methods that is being employed in the burst data analysis is based on time-frequency decomposition of detector data. These algorithms construct timefrequency maps of the time-series data and look for "timefrequency regions" containing excess power which are statistically unlikely to be associated with the background noise distribution [16]. Some of these algorithms are based on clustering the "time-frequency pixels" containing excess power, and applying another threshold on these clusters of pixels [17]. Time-frequency detection algorithms using basis functions other than the standard Fourier basis functions are also proposed $[18,19]$. Another class of burstdetection algorithms look for slopes or ridges in the timeseries data, or in its time-frequency representation [2022]. In general, these methods are claimed to be robust in detecting short-lived signals with minimum a priori information.

While the optimal filtering technique, along with accurate models of the waveforms, enables one to accurately estimate the physical parameters (such as masses and spins) of the GW source, the time-frequency methods, by construction, are unable to accomplish this. Instead, these algorithms try to parametrize the underlying gravitational waveforms using a set of quantities like the characteristic central frequency, duration, bandwidth, etc. The detection algorithms, implemented in the data analysis pipelines, are usually referred to as event trigger generators (ETGs).

Since current interferometric detectors are highly complex instruments, the detector output typically contains a large number of noise transients, or "glitches," of instrumental origin which cause the ETGs to generate spurious triggers. One of the main challenges in the burst data analysis is to distinguish these spurious bursts from actual GW bursts. Since the expected GW signals are unmodeled, it is practically impossible to distinguish these "false alarms" from actual GW bursts based on their signal characteristics. One way of dealing with this issue is to require that the triggers be coincident (within a time window) in multiple detectors located at different parts of the world. Although this "coincidence requirement" reduces the list of candidate triggers by a considerable amount, this does not completely cure the problem. While coincident instrumental bursts in multiple detectors are highly improbable, long data-taking runs (typically several months long) using multiple detectors can produce a large number of random coincidences (potentially thousands per month [23]). It is thus very important to develop robust techniques to distinguish between true GW bursts and spurious instrumental bursts which are coincident in different detectors-popularly known as veto techniques.

Since a number of environmental and instrumental noise sources can potentially couple to the main detector output, many such noise sources are continuously recorded along with the data from the main detector output. The measurement points for time-series data within the detector are referred to as "channels." One class of veto methods is based on identifying triggers in the "gravitational-wave channel" (the main detector output) which are coincident with triggers in an instrumental/environmental noise channel. The "coincidence windows" are chosen such that the "accidental" (random) coincidence rate between the two channels is limited to an acceptable amount. See [23-26] for some recent work on such "statistical vetoes." Another class of "physical vetoes" is based on our understanding of how a GW should (or should not) appear in certain channels [27-29]. Moreover, a number of "waveform consistency tests" between multiple detectors are also employed in the burst searches [30-34].

In this paper, we demonstrate a veto strategy which makes use of our understanding of the physical coupling of various detector subsystems to the detector output. This method is different from the physical veto methods discussed above, in the sense that here we use the knowledge of the coupling mechanism involved in transporting glitches from a particular subsystem to the main detector output. The main idea behind this method is that the noise in an instrumental channel $X$ can be transferred into the detector output (channel $H$ ) using the transfer function from $X$ to $H{ }^{1}$ If a noise transient in channel $H$ is causally related to one in channel $X$, the two have to be consistent with the transfer function. The basic idea of this method was formulated in [35], which also demonstrated this in the presence of stationary Gaussian noise. Here, we demonstrate a more general formulation which admits nonGaussian tails in the noise distribution and other "reallife effects" (see Sec. II C). We also propose an alternative statistic to test the consistency of the two triggers (see

\footnotetext{
${ }^{1}$ Throughout this paper, channel $X$ refers to the measurement point for time-series data from a detector subsystem/environmental noise source $X$, and channel $H$ refers to the main detector output (the "GW channel").
} 
Sec. II B). Section II reviews the basic ideas of this method. In Sec. III, we demonstrate the method by performing hardware injections in the GEO 600 detector [3]. The results of the veto analysis performed on 5 days of data from the fifth science run of GEO 600 are discussed in Sec. IV. In Sec. V, we summarize our main findings.

\section{VETOES USING KNOWN INSTRUMENTAL COUPLINGS}

Let $x_{i}$ and $h_{i}$ denote the discretely sampled time-series data recorded in channel $X$ (which records the noise from a detector subsystem) and channel $H$ (the "gravitationalwave channel"), respectively. We denote the corresponding discrete Fourier transforms (DFT) by $\tilde{x}_{k}$ and $\tilde{h}_{k}$, respectively. The input and output of a linear, time-invariant system are related by the transfer function $T_{k}$ of the system, which is defined as

$$
T_{k}^{\mathrm{XH}} \equiv \frac{P_{k}^{\mathrm{XH}}}{P_{k}^{\mathrm{XX}}}
$$

Here $P_{k}^{\mathrm{XH}} \equiv \overline{\tilde{x}_{k} \tilde{h}_{k}^{*}}$ is the cross-power spectral density of $x_{i}$ and $h_{i}$, and $P_{k}^{\mathrm{XX}} \equiv \overline{\tilde{x}_{k} \tilde{x}_{k}^{*}}$ is the power spectral density of $x_{i}$, where the "bars" indicate ensemble averages. If the coupling of noise between channel $X$ and $H$ is linear and the transfer function is time invariant, the Fourier transform of the noise measured in channel $X$ at any time can be transferred to channel $H$, by using the transfer function

$$
\tilde{x}_{k}^{\prime}=\tilde{x}_{k} T_{k}^{\mathrm{XH}} \text {. }
$$

$\tilde{x}_{k}$ and $\tilde{h}_{k}$ can be thought of as components of two vectors $\tilde{\mathbf{x}}$ and $\tilde{\mathbf{h}}$ defined in two $N$-dimensional linear vector spaces. In the mathematical sense, Eq. (2.2) maps $\tilde{\mathbf{x}}$ to the space of $\tilde{\mathbf{h}}$. In the physical sense, this means that, if a noise transient originates in $X$, one can predict how it will appear in $H$. If there exists a noise transient in $H$ at the same time, ${ }^{2}$ we can compare it with the above "prediction." If a noise transient in channel $H$ is causally related to one in channel $X$, the data vectors $\tilde{\mathbf{x}}$ and $\tilde{\mathbf{h}}$ have to be consistent with the transfer function. This allows us to formulate a powerful strategy to veto noise transients originating within the detector.

The basic idea is the following: first, we identify timecoincident burst triggers in channels $X$ and $H$. We compute the DFTs of two short segments of data in channels $X$ and $H$. The length of these segments (typically a few tens of milliseconds) is chosen so as to encompass only the noise transient under investigation. If these two noise vectors are consistent with the transfer function, as given by Eq. (2.1), it is highly likely that the noise transient originates in $X$,

\footnotetext{
${ }^{2}$ The time-coincidence window should be chosen according to the typical time scale of the transients that we are concerned with. We use a time window of a few tens of milliseconds since the current searches for $\mathrm{GW}$ bursts seek to detect bursts of duration $\ll 1 \mathrm{sec}$.
}

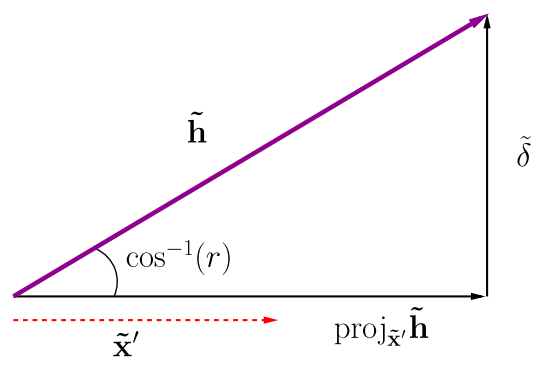

FIG. 1 (color online). Schematic diagram of the linear vector space in which the analysis methods are formulated.

and we veto the trigger. On the other hand, if the two noise vectors are inconsistent with the transfer function, we conclude that this particular noise transient in $H$ does not have its origin in $X$. In the following subsections, we construct two different statistics which can be used to make this decision. One statistic is based on constructing a null stream between channel $H$ and the "transferred" channel $X$; i.e., if the noise transient originates in subsystem $X$, and is sensed by channel $X$, it is possible to construct a linear combination of the two data streams such that it does not contain any excess power. The second statistic is based on the cross correlation of the noise in channel $H$ with the "transferred" noise in channel $X$.

In the following two subsections, we assume that the data streams $x_{i}$ and $h_{i}$ are drawn from zero-mean Gaussian distributions. Also, we assume that the transfer function from $X$ to $H$ is accurately measured/calculated and is time invariant. In the third subsection, we discuss the "real-life scenario" where the data streams are not perfectly Gaussian and the transfer function is nonstationary.

The linear vector space in which the analysis methods are formulated is schematically illustrated in Fig. 1.

\section{A. Using the null stream}

The null stream between $\tilde{\mathbf{h}}$ and $\tilde{\mathbf{x}}^{\prime}$ is the component of the vector $\tilde{\mathbf{h}}$ orthogonal to $\tilde{\mathbf{x}}^{\prime}$. This can be constructed using the Gram-Schmidt orthogonalization [36]:

$$
\tilde{\boldsymbol{\delta}}=\tilde{\mathbf{h}}-\operatorname{proj}_{\tilde{\mathbf{x}}^{\prime}} \tilde{\mathbf{h}},
$$

where we define the projection operator by

$$
\operatorname{proj}_{\tilde{\mathbf{u}}} \tilde{\mathbf{v}}=\frac{\langle\tilde{\mathbf{v}}, \tilde{\mathbf{u}}\rangle}{\langle\tilde{\mathbf{u}}, \tilde{\mathbf{u}}\rangle} \tilde{\mathbf{u}} .
$$

In the above expression $\langle\tilde{\mathbf{v}}, \tilde{\mathbf{u}}\rangle$ denotes the inner product between the vectors $\tilde{\mathbf{v}}$ and $\tilde{\mathbf{u}}$ :

$$
\langle\tilde{\mathbf{v}}, \tilde{\mathbf{u}}\rangle=\operatorname{Re} \sum_{k} \tilde{\boldsymbol{v}}_{k} \tilde{u}_{k}^{*}
$$

If the noise transient originates in $X$, it will completely disappear in $\tilde{\boldsymbol{\delta}}$. In order to test this, we compute the excesspower statistic [16] from $\tilde{\boldsymbol{\delta}}$ : 


$$
\epsilon_{\delta}=\sum_{k} \frac{\left|\tilde{\delta}_{k}\right|^{2}}{\sigma_{k}^{2}},
$$

where $\sigma_{k}^{2}$ is the expected variance of $\tilde{\delta}_{k}$ in the absence of any excess power. In the absence of any excess power in $\tilde{\boldsymbol{\delta}}$, $\epsilon_{\delta}$ will follow a Gamma distribution [37]. The scale parameter $\alpha$ and shape parameter $\beta$ can be estimated from the stationary noise (see [35] for more details).

If $\epsilon_{\delta}$ is less than, or equal to, a threshold $\tau$, we veto the trigger. The threshold $\tau$ giving a rejection probability (probability that a "causal" trigger is vetoed) $\Phi$ can be calculated from

$$
\Phi=\int_{0}^{\tau} \Gamma(x ; \alpha, \beta) \mathrm{d} x,
$$

where $\Gamma(x ; \alpha, \beta)$ is the probability density of the Gamma distribution with parameters $\alpha$ and $\beta$.

\section{B. Using the cross correlation}

The linear cross-correlation coefficient between two vectors $\tilde{\mathbf{x}}^{\prime}$ and $\tilde{\mathbf{h}}$ is the cosine of the angle between them:

$$
r=\operatorname{Re} \frac{\left\langle\tilde{\mathbf{x}}^{\prime}, \tilde{\mathbf{h}}\right\rangle}{\left\|\tilde{\mathbf{x}}^{\prime}\right\|\|\tilde{\mathbf{h}}\|},
$$

where $\|\mathbf{u}\|$ denotes the magnitude of the vector $\mathbf{u}$. If the noise transient in channel $H$ indeed originates in $X, \tilde{\mathbf{x}}^{\prime}$ and $\tilde{\mathbf{h}}$ should display a high correlation. On the other hand, if the noise transient does not originate in $X$, the vectors $\tilde{\mathbf{x}}^{\prime}$ and $\tilde{\mathbf{h}}$ will be randomly oriented, and hence the linear cross-correlation coefficient $r$ will tend to be small in absolute value. This can be converted to the normally distributed variable $z$ by the Fisher transformation [38]:

$$
z=\frac{1}{2} \ln \left(\frac{1+r}{1-r}\right)
$$

The new variable $z$ will be approximately normally distributed with mean zero and standard deviation $1 / \sqrt{N-3}$, where $N$ is the dimension of the vectors $\tilde{\mathbf{x}}^{\prime}$ and $\tilde{\mathbf{h}}$.

If $z$ is greater than, or equal to, a threshold $\lambda$, we veto the trigger. The threshold giving an accidental veto probability of $\psi$ can be calculated from

$$
\psi=\int_{\lambda}^{\infty} f\left(x ; \mu, \sigma^{2}\right) \mathrm{d} x,
$$

where $f\left(x ; \mu, \sigma^{2}\right)$ is the probability density of the normal distribution with mean $\mu=0$ and variance $\sigma^{2}=1 /(N-$ 3).

\section{Implementation}

The assumption we made in the previous subsections that the transfer function is time invariant is strictly not true. Transfer functions in actual detectors can vary in time. The slow temporal variation of the transfer function can be taken into account by making repeated measurements of the transfer function and tracking its evolution by continuously injecting and measuring spectral lines at certain frequencies (see [39]). But the nonstationarities of the transfer function on short time scales are hard to track. It may also be noted that the noise in the present-generation interferometers is not stationary Gaussian and exhibits tails in the distribution. Considering these "real-life" effects, it may not be wise to use the "ideal-case" relations given by Eqs. (2.7) and (2.10) to compute the veto thresholds. For instance, due to the imperfect transfer function, the "null stream" $\tilde{\boldsymbol{\delta}}$ can contain some "residual burst," and, as a result, the excess-power statistic computed from $\tilde{\boldsymbol{\delta}}$ will not fall into the expected Gamma distribution. But, we do expect the excess-power statistic $\epsilon_{\delta}$ computed from $\tilde{\boldsymbol{\delta}}$ to be smaller than the same $\left(\epsilon_{\mathrm{h}}\right)$ computed from $\tilde{\mathbf{h}}$. If the ratio $s \equiv \epsilon_{\mathrm{h}} / \epsilon_{\delta}$ is greater than a threshold, we veto the trigger. The veto threshold corresponding to a certain accidental veto probability is calculated as described below.

We time shift $x_{i}$ with respect to $h_{i}$ to destroy the causal relationship between the two data streams. ${ }^{3}$ The coincident triggers in the time-shifted data streams are identified and the "excess-power ratio," $s$, for each coincident trigger is calculated. $n$ such time shifts are performed to get better statistics from the data. A threshold, $\tau_{s}$, is chosen such that only an acceptable number of coincident triggers in the time-shifted analysis have $s \geq \tau_{s}$. This threshold $\tau_{s}$ is used to veto the triggers in the "zero-lag" analysis (without time shifting the data). The time-shifted analysis can also be used to calculate the veto threshold $\lambda$ for the analysis using the cross-correlation statistic. Here, we choose a threshold $\lambda$ such that only an acceptable number of coincident triggers have $z \geq \lambda$ in the time-shifted analysis and use this threshold to do the zero-lag analysis. The veto pipeline is schematically illustrated in Fig. 2.

\section{Caveats}

It is worth stressing that this method relies on the linearity in the coupling of the noise from the detector subsystem $X$ to the detector output, and cannot be used where the coupling is nonlinear. This method also assumes that the transfer function from $X$ to $H$ is unique, and channel $X$ accurately senses the disturbances in $X$. In other words, this technique can only be applied to systems that exhibit a linear coupling through a set path, or multiple paths that are fixed. An environmental monitor will often fail to meet this requirement, unless the sensors are exceptionally well placed, because each local disturbance could couple differently to the monitor and the GW channels,

\footnotetext{
${ }^{3}$ Time-shift analysis is commonly employed in burst searches in order to estimate the accidental consistency, or "background" rate. See, for example, [23].
} 


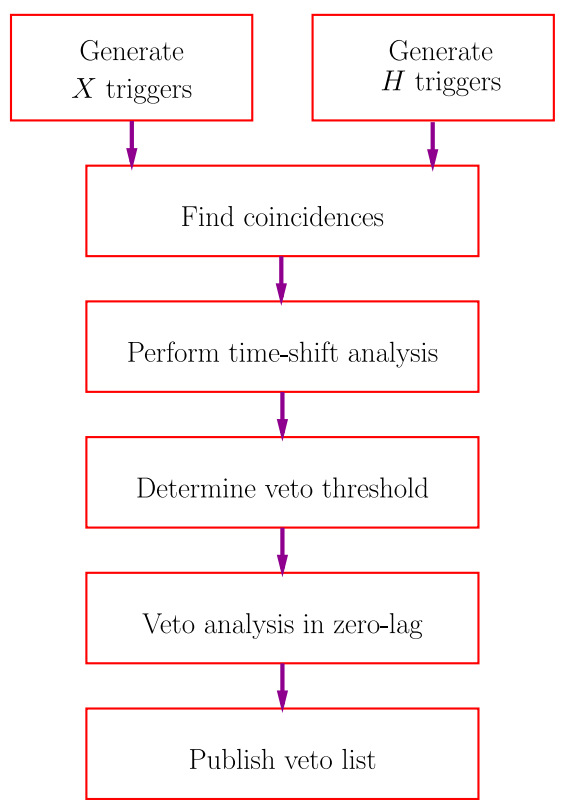

FIG. 2 (color online). Schematic illustration of the veto pipeline.

meaning that a different transfer function would be needed for each physical point of origin for the disturbance.

\section{ANALYSIS ON HARDWARE-INJECTED BURST SIGNALS}

\section{A. Injections mimicking instrumental bursts}

In order to test this veto method, we injected around 300 sine-Gaussian burst signals over a period of 1 hour into four subsystems of GEO 600, whose couplings to $H$ were known and well understood; the injections were performed serially, one subsystem after another. The four subsystems we chose are listed below. These descriptions are technical and concise. For more information, refer to [40].

Laser amplitude noise $\left(P_{\text {ref }}\right)$. - We make bursts of laser amplitude noise by injecting glitches into the laser amplitude stabilization loop. We detect these glitches by measuring the light power reflected from the power-recycling cavity in the data acquisition system as channel $P_{\text {ref }}$.

Laser frequency noise $\left(F_{\text {ref }}\right)$. - We make bursts of laser frequency noise by adding glitches to the error point of the Michelson common-mode control servo which keeps the power-recycling cavity resonant by adjusting the frequency of the master laser. The recording of this error point in the data acquisition system serves as the veto channel, $F_{\text {ref }}$.

Michelson oscillator phase noise $\left(\Phi_{\mathrm{MI}}\right)$.- The Michelson differential arm length in GEO 600 is controlled by imposing phase-modulation sidebands on the light entering the interferometer. A heterodyne readout scheme is then used to derive an error signal which is fed back to the end mirrors of the Michelson to keep it on a dark fringe. We make glitches in the phase of the oscillator used to add the modulation sidebands by driving the voltage-frequency-control input of the crystal oscillator used to create this modulation signal. We phase lock a reference crystal oscillator to the main crystal oscillator, and the error point of the phase-locked loop, which is sensitive to phase fluctuations on both oscillator signals, is recorded in the data acquisition system as $\Phi_{\mathrm{MI}}$ and serves as a sensitive measurement of the phase noise on the main Michelson modulation sidebands.

Michelson oscillator amplitude noise $\left(A_{\mathrm{MI}}\right)$. - The amplitude of the main crystal oscillator is also stabilized to a quiet DC reference. We can add signals to the error point of this stabilization servo so as to impose additional amplitude noise on the main Michelson modulation signal. We added glitch signals into this control loop and recorded its error point in the data acquisition system as $A_{\mathrm{MI}}$ to serve as a veto channel.

Burst triggers in the veto channel and the GW channel are generated using the mHACR [25,35] burst-detection algorithm. mHACR belongs to the class of time-frequency detection algorithms that make a time-frequency map of the data and identify time-frequency pixels containing excess power which are statistically unlikely to be associated with the underlying noise distribution. For a detailed description of the algorithm and its performance, see [25]. However, we remind the reader that the details of the burstdetection algorithm are immaterial as far as this veto method is concerned. The burst ETG is only used to identify the coincident triggers in the two channels, and any ETG with proper time estimation of the burst event should serve this purpose.

Coincident triggers in the two channels are identified using a time window of $\pm 10 \mathrm{~ms}$. The results of the timeshift analysis on the hardware injections performed in the $\Phi_{\mathrm{MI}}$ are shown in Figs. 3 and 4. The horizontal axis shows the time shift applied between $x_{i}$ and $h_{i}$. The vertical axis in Fig. 3 shows the excess-power ratio $s \equiv \epsilon_{\mathrm{h}} / \epsilon_{\delta}$. The (black) dots correspond to the coincident triggers in the time-shifted analysis, and the (red) crosses correspond to the ones in the zero-lag analysis. From this, a veto threshold of 2.35 is chosen which corresponds to an accidental veto rate of 1 per day. All the coincident triggers in the zero-lag analysis are vetoed using this threshold. The vertical axis in Fig. 4 shows the (transformed) crosscorrelation statistic $z$. The (black) dots correspond to the coincident triggers in the time-shifted analysis, and the (blue) crosses correspond to the ones in the zero-lag analysis. The veto threshold corresponding to an accidental veto rate of 1 per day is 0.27 , which resulted in vetoing $99 \%$ of the coincident triggers in the zero-lag analysis.

The veto analysis is performed on all four channels in which the hardware injections are done. Results of the analysis are summarized in Table I. It can be seen that only $\sim 5 \%$ of the coincident triggers in the time-shifted analysis are vetoed, while more than $90 \%$ of the coincident 


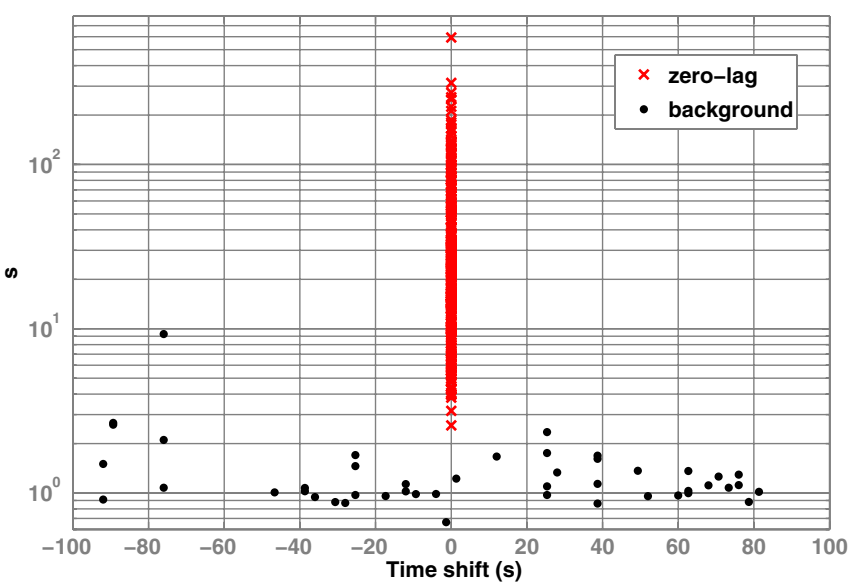

FIG. 3 (color online). Time-shift analysis on "instrumentalglitch-like" hardware injections performed in the $\Phi_{\mathrm{MI}}$ channel. The horizontal axis shows the time shift applied between $x_{i}$ and $h_{i}$, and the vertical axis shows the excess-power ratio $s$. The (black) dots correspond to the coincident triggers in the timeshifted analysis, and the (red) crosses correspond to the ones in the zero-lag analysis.

triggers in the zero-lag analysis are vetoed. This implies that the accidental rate of the veto is only $\sim 5 \%$ of that of the standard statistical veto (using a time window of $\pm 10 \mathrm{~ms}$ ) for almost the same veto efficiencies.

\section{B. Injections mimicking gravitational-wave bursts}

Some of the interferometer channels are sensitive to GWs to some non-negligible level. This raises the question of veto safety while using interferometer channels as veto channels; i.e., we have to make sure that we do not veto actual GW bursts which are coincident in the two channels. We argue that, since actual GW bursts are not causally

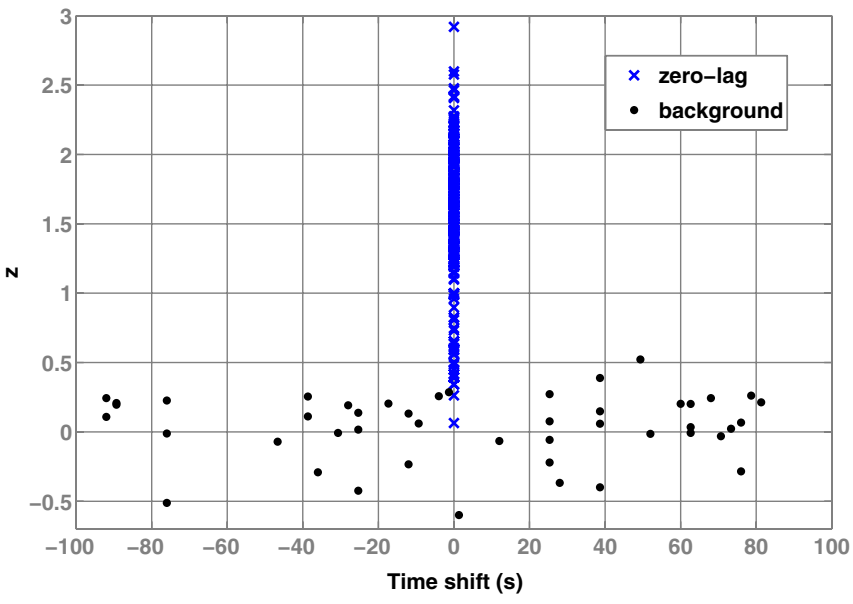

FIG. 4 (color online). Same as in Fig. 3, except that the vertical axis shows the cross-correlation statistic $z$. The (black) dots correspond to the coincident triggers in the time-shifted analysis, and the (blue) crosses correspond to the ones in the zero-lag analysis.
TABLE I. Summary of the veto analysis on hardware injections mimicking instrumental bursts. $\tau_{s}$ and $\lambda$ are the chosen veto thresholds on the excess-power ratio $s$ and the crosscorrelation statistic $z$, respectively. $\xi$ is the fraction of coincident events that are vetoed in the time-shifted analysis. The fraction of coincident events vetoed in the zero-lag analysis using the $s$ statistic is denoted by $\chi_{s}$, while the same using the $z$ statistic is denoted by $\chi_{z}$. The chosen thresholds correspond to an accidental veto rate of 1 per day.

\begin{tabular}{lccccc}
\hline \hline Veto channel & \multicolumn{2}{c}{ Threshold } & \multicolumn{3}{c}{ Veto fraction } \\
& $\tau_{s}$ & $\lambda$ & $\xi$ & $\chi_{s}$ & $\chi_{z}$ \\
\hline$F_{\text {ref }}$ & 2.51 & 0.33 & $4.48 \times 10^{-2}$ & 0.90 & 0.90 \\
$P_{\text {ref }}$ & 1.94 & 0.23 & $5.45 \times 10^{-2}$ & 1.00 & 1.00 \\
$\Phi_{\text {MI }}$ & 2.35 & 0.27 & $6.12 \times 10^{-2}$ & 1.00 & 0.99 \\
$A_{\mathrm{MI}}$ & 1.50 & 0.26 & $4.62 \times 10^{-2}$ & 0.97 & 0.97 \\
\hline \hline
\end{tabular}

related to the instrumental channels, the coincident triggers in channels $X$ and $H$ will not be consistent with the transfer function from $X$ to $H$ and, hence, will not be vetoed using this method. Although the four channels under investigation in this paper are not expected to show any nonnegligible sensitivity to GWs, there can be unexpected couplings, for example, through electrical faults or cross couplings in the data acquisition system. It is therefore prudent to explicitly demonstrate the safety of this veto method by doing GW-like hardware injections.

Hardware injections are performed by injecting signals into the electrostatic actuators used to control the differential-arm-length degree of freedom of GEO 600. For the test described here, around 300 sine-Gaussian bursts were injected with varying amplitudes and with central frequencies in the range 200 to $1300 \mathrm{~Hz}$.

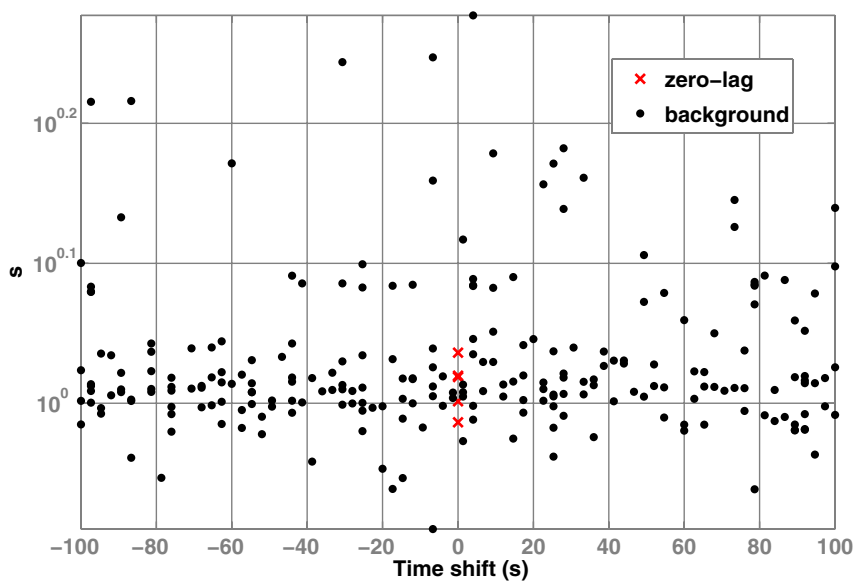

FIG. 5 (color online). Time-shift analysis on the GW-like hardware injections. $E_{\text {ref }}$ is used as the veto channel. The horizontal axis shows the time shift applied between $x_{i}$ and $h_{i}$, and the vertical axis shows the excess-power ratio $s$. The (black) dots correspond to the coincident triggers in the time-shifted analysis, and the (red) crosses correspond to the ones in the zerolag analysis. 


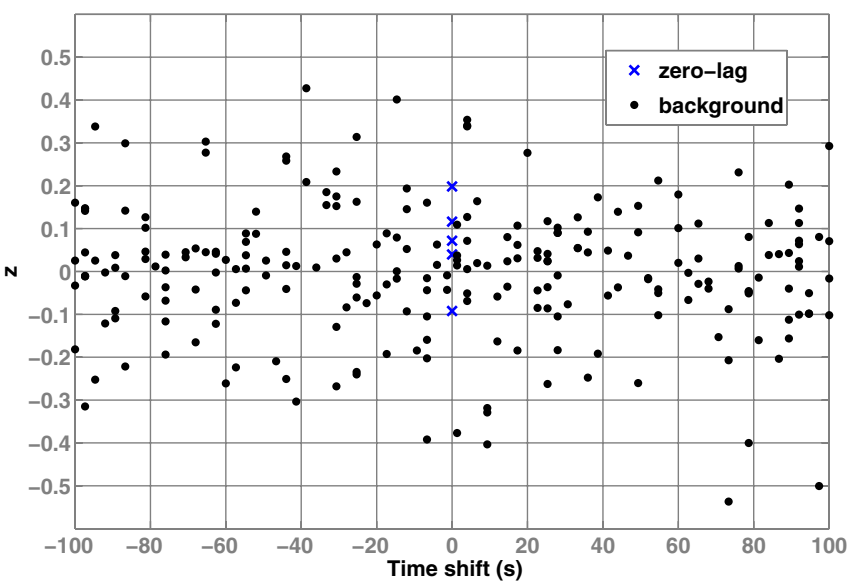

FIG. 6 (color online). Same as in Fig. 5, except that the vertical axis shows the cross-correlation statistic $z$. The (black) dots correspond to the coincident triggers in the time-shifted analysis, and the (blue) crosses correspond to the ones in the zero-lag analysis.

Figures 5 and 6 show the results of the veto analysis performed on the GW-like hardware injections. $F_{\text {ref }}$ is used as the veto channel. It can be seen that neither of the test statistics $(s$ or $z)$ in the zero-lag analysis shows any excess significance over the corresponding time-shifted analysis. The veto thresholds corresponding to an accidental rate of 1 per day are $\tau_{\mathrm{s}}=1.64$ and $\lambda=0.35$. Using these thresholds, we do not veto any of the injections.

\section{AN EXAMPLE APPLICATION}

GEO 600 participated full time in the "fifth science run" (S5 run) in coincidence with the LIGO detectors from May 2006 to October 2006. The first few weeks of $H$ data contained an additional population of glitches identified as coming from the laser frequency stabilization control loop. These excess glitches had central frequencies typically around $2 \mathrm{kHz}$. In fact, the glitches were broadband in the frequency stabilization loop, but the coupling of frequency noise to $H$ is most prominent around $2 \mathrm{kHz}$ and so this is where we see the excess noise in $H$. The identification and repair of the source of these glitches took several weeks. So we identified an appropriate measure $\left(F_{\text {ref }}\right)$ of the frequency-noise glitches that could be used as a veto channel (see Sec. III A to see how this channel is derived).

Veto analysis is performed on 5 days of data from the period described above (in the frequency range of $400 \mathrm{~Hz}-$ $2 \mathrm{kHz}$ ). Burst triggers in the two channels are generated by the mHACR burst-detection algorithm. Coincident triggers within the two channels are identified using a time window of $\pm 10 \mathrm{~ms}$ for time coincidence. Only triggers with a signal-to-noise ratio $\geq 6$ are considered for this analysis. Out of 5326 triggers in the GW channel, 2048 triggers were found to be coincident with the $F_{\text {ref }}$ channel. The accidental rate of the veto is estimated by doing 76 time shifts (from $-100 \mathrm{sec}$ to $100 \mathrm{sec}$ ). Figure 7 shows the excesspower ratio $s$ computed from the coincident triggers plotted against the applied time shift between the data streams, while Fig. 8 shows the cross-correlation statistic $z$ plotted against the time shift. The dots correspond to the coincident triggers from the time-shifted analysis, and the crosses correspond to those from the zero-lag analysis. We choose an accidental veto rate of 1 per day. The thresholds on the two statistics are estimated from the time-shifted analysis. This corresponds to a threshold of $\tau_{\mathrm{s}}=2.25$ for the excess-power ratio $s$ and a threshold of $\lambda=0.54$ for the cross-correlation statistic $z$. In the analysis using the null stream, all coincident triggers with $s \geq \tau_{\mathrm{s}}$ are vetoed, while in the analysis using the cross-correlation statistic, all triggers with $z \geq \lambda$ are vetoed. It was found that $88 \%$ of the coincident triggers are vetoed using the null-stream method and $92 \%$ of the coincident triggers are vetoed using the cross-correlation method. These correspond to 34\% and $35 \%$ of the total number of $H$ triggers in the data.

Histograms of the two test statistics $s$ and $z$ computed from the coincident triggers are plotted in Figs. 9 and 10. The plots on the left show the distributions of the test statistics computed from the time-shifted analysis, normalized by the number of time shifts applied. These are the expected distributions of $s$ and $z$ in the absence of any causal relation between triggers in $X$ and $H$ (for the given data set). Histograms on the right show the distributions of $s$ and $z$ computed from the zero-lag analysis. Two different populations are clearly visible in these plots. One population (centered around 1 in the histograms of $s$; centered around zero in the histograms of $z$ ) corresponds to the triggers which are accidentally time coincident in the channels $X$ and $H$, while the other population (centered

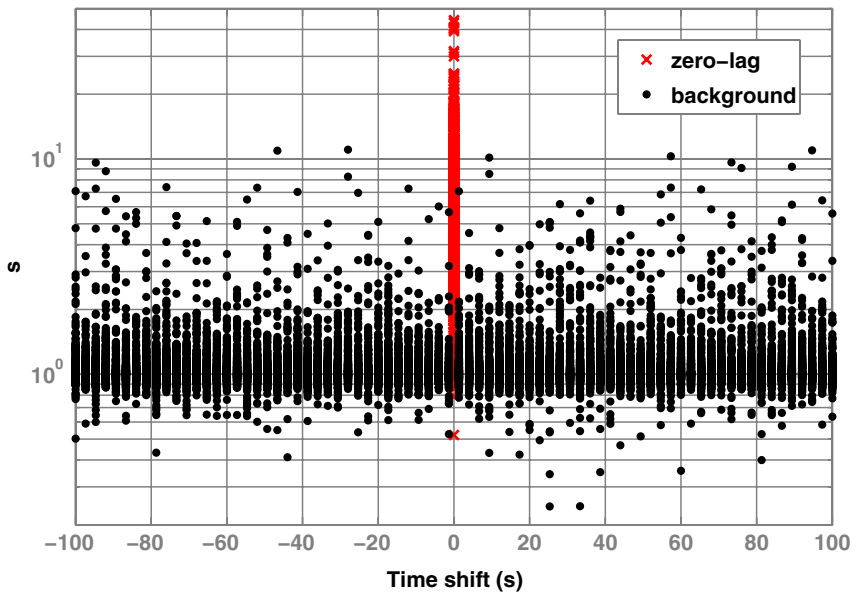

FIG. 7 (color online). Time-shifted analysis on 5 days of data from the fifth science run of GEO 600 using $F_{\text {ref }}$ as the veto channel. The horizontal axis shows the time shift applied between $x_{i}$ and $h_{i}$, and the vertical axis shows the excess-power ratio $s$. The (black) dots correspond to the coincident triggers in the time-shifted analysis, and the (red) crosses correspond to the ones in the zero-lag analysis. 


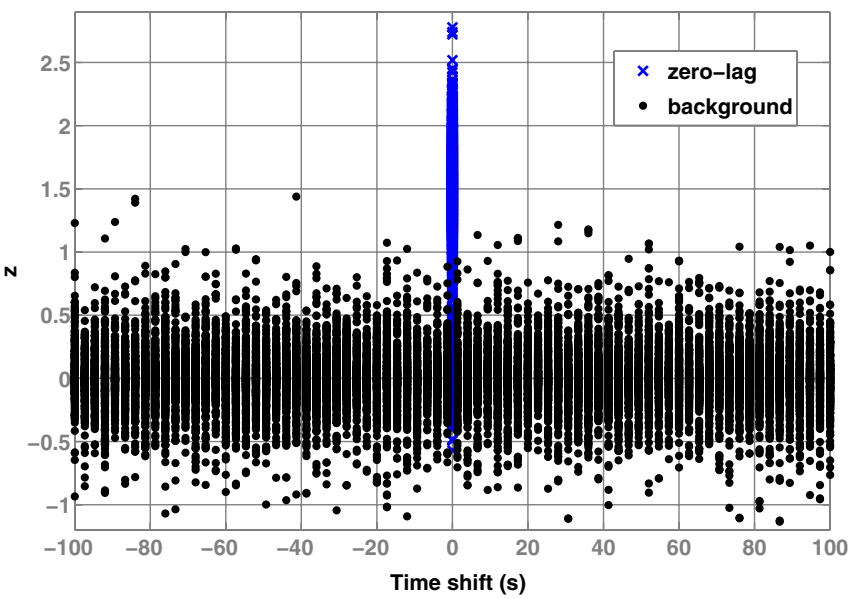

FIG. 8 (color online). Same as in Fig. 7, except that the vertical axis shows the cross-correlation statistic $z$. The (black) dots correspond to the coincident triggers in the time-shifted analysis, and the (blue) crosses correspond to the ones in the zero-lag analysis.

around 6 in the histogram of $s$; centered around 1.6 in the histogram of $z$ ) corresponds to triggers in $H$ which are causally related to the ones in $X$. It is interesting to note that this method is able to distinguish clearly between these two populations. The reader may note that the number of
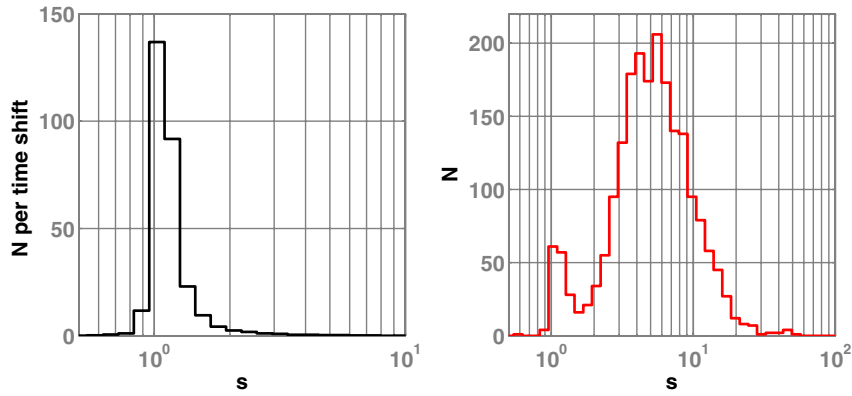

FIG. 9 (color online). Histograms of the excess-power ratio $s$ computed from the time-shifted analysis (left panel) and the zero-lag analysis (right panel).
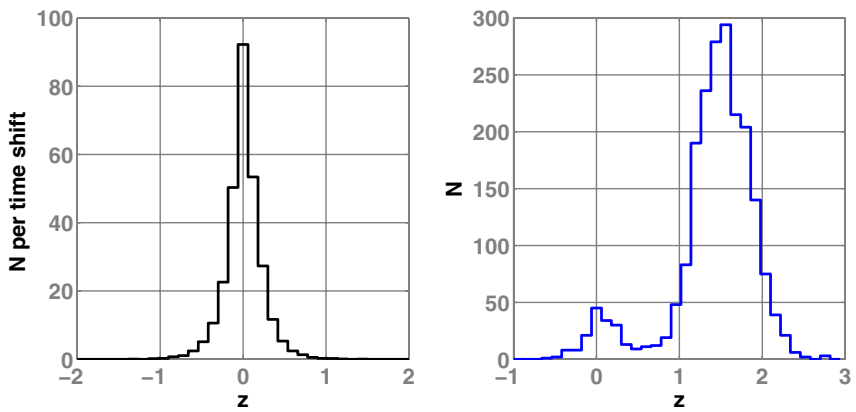

FIG. 10 (color online). Histograms of the cross-correlation statistic $z$ computed from the time-shifted analysis (left panel) and the zero-lag analysis (right panel). accidental coincidences in the time-shifted analysis is $\sim 35 \%$ larger than that in the zero-lag analysis. This can be explained in the following way: the veto analysis has shown that around $35 \%$ of the triggers in channel $H$ are causally related to $F_{\text {ref }}$. These "causal" triggers will fall into the populations on the right in the zero-lag analysis (centered around 6 in the histograms of $s$; centered around 1.6 in the histograms of $z$ ). But, since the accidental coincidence rate is directly proportional to the total number of triggers, the presence of these causal triggers in the data would increase the coincidence rate in the time-shifted analysis by $\sim 35 \%$, thus explaining the excess coincidences that we observe.

Figure 11 shows a time-frequency plot of the mHACR triggers from 5 days of GEO 600 data. The (green) circles correspond to the coincident triggers (in channel $H$ ) which are vetoed using the null-stream method, and the (black) dots correspond to the ones which are vetoed using the cross-correlation method. The (red) crosses correspond to the coincident triggers which are not vetoed by any of the methods.

A summary of the analyses performed using different accidental veto rates is given in Table II. Also, in Fig. 12, we plot the fraction of coincident events that are vetoed in the zero-lag analysis (a measure of the efficiency of the veto) against the fraction of the coincident triggers which are vetoed in the time-shifted analysis (a measure of the accidental veto probability). This plot can be thought of as a receiver operating characteristic [41] plot for this analysis, and can be used to choose thresholds which correspond

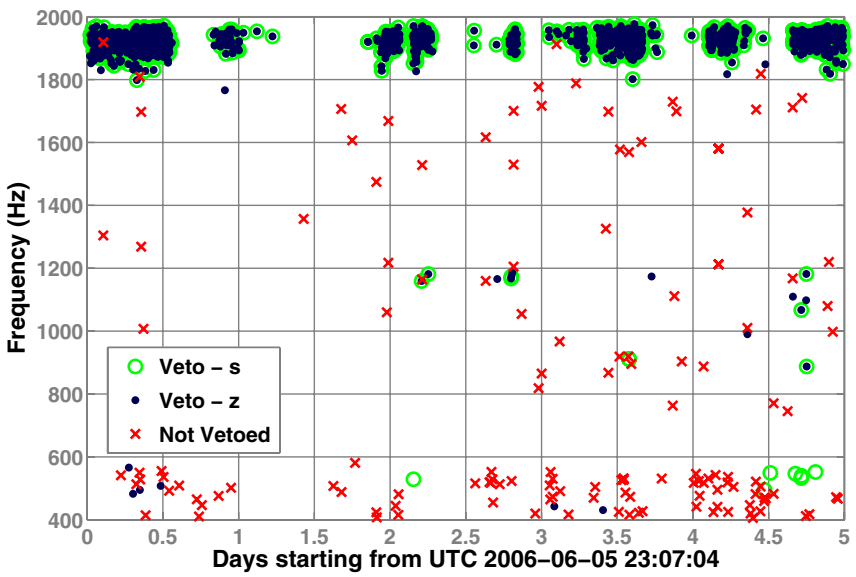

FIG. 11 (color online). A time-frequency plot of mHACR triggers from 5 days of GEO 600 data. The horizontal axis shows the time and the vertical axis shows the frequency of the burst triggers in channel $H$, as estimated by mHACR. Only those triggers which are coincident with $F_{\text {ref }}$ are plotted. The (green) circles correspond to coincident triggers which are vetoed using the null-stream method, and the (black) dots correspond to the ones which are vetoed using the crosscorrelation method. Coincident triggers which are not vetoed by any of the methods are indicated by (red) crosses. The chosen veto thresholds correspond to one accidental veto per day. 
TABLE II. Summary of the veto analysis on 5 days of data from GEO 600. $\tau_{s}$ and $\lambda$ are the chosen veto thresholds on the excess-power ratio $s$ and the cross-correlation statistic $z$, respectively. $\xi$ is the fraction of coincident events that are vetoed in the time-shifted analysis. The fraction of coincident events vetoed in the zero-lag analysis using the $s$ statistic is denoted by $\chi_{s}$, while the same using the $z$ statistic is denoted by $\chi_{z}$. The chosen veto thresholds correspond to the accidental veto rates tabulated in the first column.

\begin{tabular}{lccccc}
\hline \hline \multirow{2}{*}{ Accidental rate } & \multicolumn{2}{c}{ Threshold } & \multicolumn{3}{c}{ Veto fraction } \\
& $\tau_{s}$ & $\lambda$ & $\xi$ & $\chi_{s}$ & $\chi_{z}$ \\
\hline day $^{-1}$ & 2.25 & 0.54 & $1.73 \times 10^{-2}$ & 0.88 & 0.92 \\
week $^{-1}$ & 5.09 & 0.86 & $2.47 \times 10^{-3}$ & 0.45 & 0.90 \\
month $^{-1}$ & 7.40 & 1.11 & $5.93 \times 10^{-4}$ & 0.23 & 0.85 \\
\hline \hline
\end{tabular}

to acceptable values of veto efficiency and accidental veto rate/probability. In the figure, the solid curve corresponds to the analysis using the null stream and the dashed curve corresponds to the analysis using cross correlation. It can be seen that, for high values of accidental veto probability, the two methods perform equally well. But for low values of accidental veto probability $\left(<2 \times 10^{-2}\right)$, the curve corresponding to the null-stream analysis starts to fall off, and the cross-correlation analysis continues to perform well.

Figure 13 provides a rough comparison between the abilities of the two test statistics ( $s$ and $z$ ) in vetoing the

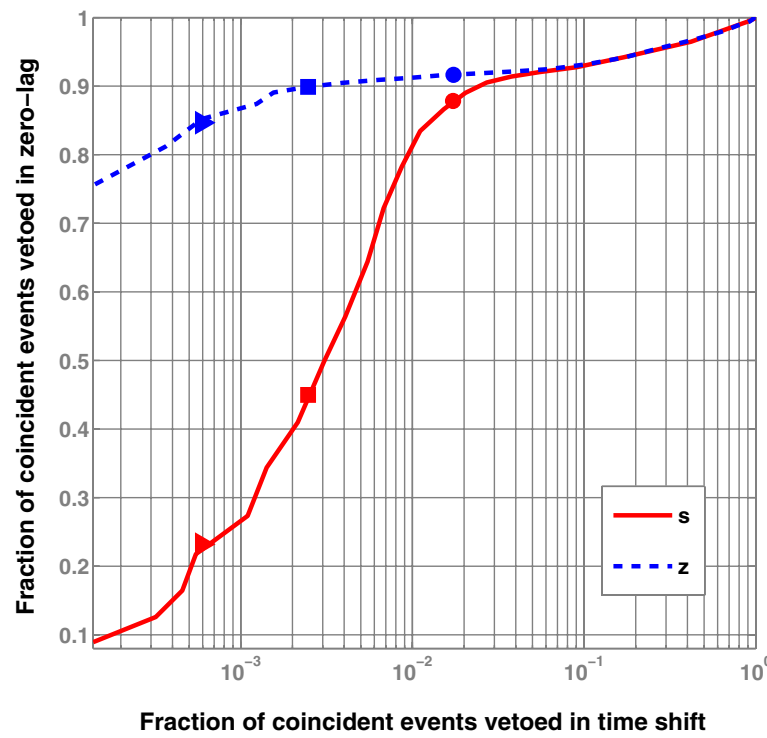

FIG. 12 (color online). Fraction of coincident triggers that are vetoed in the zero-lag analysis plotted against the fraction of the coincident triggers that are vetoed in the time-shifted analysis. The solid curve corresponds to the analysis using the null stream, and the dashed curve corresponds to the analysis using cross correlation. The triangles, squares, and dots correspond to accidental veto rates of 1 per month, 1 per week, and 1 per day, respectively. instrumental glitches. The horizontal axis shows the excess-power ratio $s$, and the vertical axis shows the cross-correlation statistic $z$ computed from the coincident triggers. The vertical (red) and horizontal (blue) lines in the plot correspond to the veto thresholds $\tau_{s}$ and $\lambda$ on the two statistics, respectively. Triggers on the right of the vertical line are vetoed by $s$, and those above the horizontal line are vetoed by $z$. Of the total number of $H$ triggers, $33.5 \%$ are vetoed by both methods. There exists a small population ( $\sim 1 \%$ of the total number of $H$ triggers) which is vetoed by $z$, but not by $s$, which suggests that $z$ is a more sensitive statistic than $s$. But this may not be taken as a general indication that the cross correlation is a more sensitive method than the null stream. One can construct alternative statistics using the null stream, which could be more sensitive than $s$. One possible alternative is $\epsilon_{\delta} / \epsilon_{\mathrm{opt}}$, where $\epsilon_{\text {opt }}$ is the excess-power statistic computed from the optimal combination $[42,43]$ of the noise vectors $\tilde{\mathbf{h}}$ and $\tilde{\mathbf{x}}^{\prime}$.

\section{SUMMARY AND OUTLOOK}

One of the most challenging problems in the search for unmodeled GW bursts using ground-based detectors is to distinguish between actual GW bursts and spurious instrumental bursts that trigger the detection algorithms. In this paper, we proposed a veto method which makes use of the information on the physical coupling of different detector subsystems to the main detector output. We also demonstrated this method using the data of the GEO 600 detector. By performing hardware injections mimicking instrumental glitches, we showed that glitches originating in a detector subsystem can be vetoed using the transfer function from the subsystem to the detector output. We also ad-

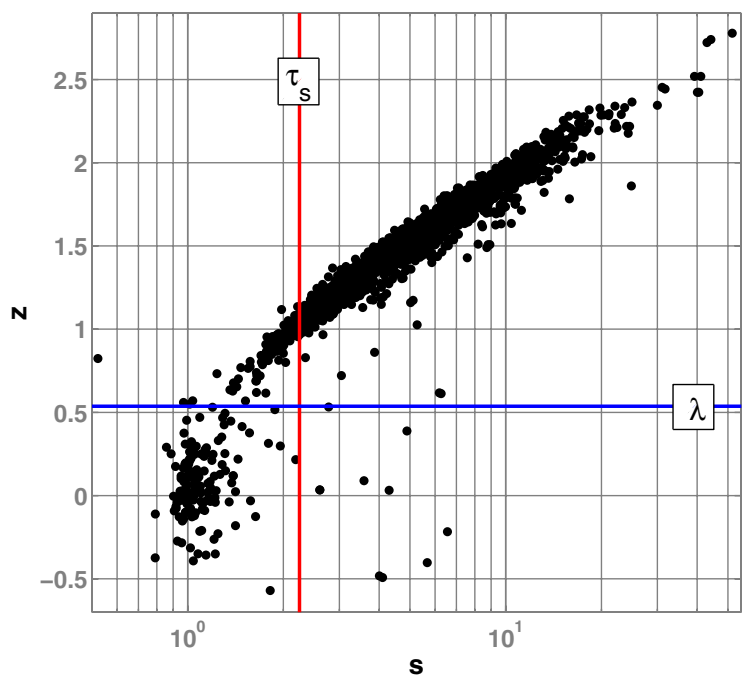

FIG. 13 (color online). Excess-power ratio $s$ (horizontal axis) computed from the coincident triggers plotted against the crosscorrelation statistic $z$ (vertical axis). The vertical (red) and horizontal (blue) lines correspond to the veto thresholds $\tau_{s}$ and $\lambda$ on the two statistics, respectively. 
dressed the issue of veto safety by performing hardware injections mimicking GW bursts into GEO 600, and by showing that such injections are not vetoed. Finally, we used this strategy to veto glitches in the data from the fifth science run of GEO 600, using the laser frequency-noise channel as the veto channel. The analysis was performed on 5 days of GEO 600 data from the second month of the science run. Thirty-five percent of the 5326 triggers in the GW channel were vetoed with an accidental rate of 1 per day using the cross-correlation method, while $34 \%$ of the triggers were vetoed using the null-stream method.

The method relies on linearity in the coupling of the noise from a detector subsystem to the detector output, and the measurability/calculability and uniqueness of the transfer function. The assumption of linear coupling is valid as far as many detector subsystems in the large-scale interferometers are concerned. Strictly speaking, this method also requires time-invariant transfer functions. The way to track down slow temporal variations in the transfer functions is discussed in the paper. The formulation that we have developed was found to be robust against nonstatio- narities of short time scales in the transfer functions, and non-Gaussian tails in the noise distribution.

When possible, using physical information has clear advantages over relying only on statistical correlations. The method that was proposed in this paper is a fully coherent way of testing the consistency of the glitches in the GW channel with those in an instrumental channel. The authors hope that this will serve as a first step for developing a class of "physical instrumental vetoes" for present and future detectors.

\section{ACKNOWLEDGMENTS}

The authors are grateful for support from PPARC and the University of Glasgow in the U.K., and the BMBF and the state of Lower Saxony in Germany. The authors also thank Peter Shawhan and Peter Saulson for their detailed comments on the manuscript, and the members of the GEO 600 group of the Albert Einstein Institute for useful discussions. This document has been assigned LIGO Laboratory Document No. LIGO-P070032-00-Z.
[1] D. Sigg (for the LIGO Scientific Collaboration), Classical Quantum Gravity 23, S51 (2006).

[2] F. Acernese et al., Classical Quantum Gravity 23, S63 (2006).

[3] H. Lück et al., Classical Quantum Gravity 23, S71 (2006).

[4] M. Ando and TAMA Collaboration, Classical Quantum Gravity 22, S881 (2005).

[5] P. Astone et al., Classical Quantum Gravity 23, S57 (2006).

[6] J.-P. Zendri et al., Classical Quantum Gravity 19, 1925 (2002).

[7] http://sam.phys.lsu.edu/.

[8] C. M. Will, Living Rev. Relativity 9, 3 (2006), http:// www.livingreviews.org/lrr-2006-3.

[9] C. Cutler, T. A. Apostolatos, L. Bildsten, L. S. Finn, E. E. Flanagan, D. Kennefick, D. M. Markovic, A. Ori, E. Poisson, G. J. Sussman, and K. S. Thorne, Phys. Rev. Lett. 70, 2984 (1993).

[10] F. D. Ryan, Phys. Rev. D 52, 5707 (1995).

[11] B. F. Schutz, Nature (London) 323, 310 (1986); Classical Quantum Gravity 6, 1761 (1989).

[12] D. Markovic, Phys. Rev. D 48, 4738 (1993).

[13] D. F. Chernoff and L. S. Finn, Astrophys. J. Lett. 411, L5 (1993).

[14] B. Allen, in Proceedings of the Spanish Relativity Meeting, 1996, edited by J.A. Miralles, J.A. Morales, and D. Saez, p. 3.

[15] C. Cutler and K.S. Thorne, in Proceedings of GR16, edited by N.T. Bishop and S.D. Maharaj (World Scientific, Singapore, 2002).

[16] W. G. Anderson, P. R. Brady, J.D. E. Creighton, and É.É. Flanagan, Phys. Rev. D 63, 042003 (2001).
[17] J. Sylvestre, Phys. Rev. D 66, 102004 (2002).

[18] S. Klimenko and G. Mitselmakher, Classical Quantum Gravity 21, S1819 (2004).

[19] S. Chatterji, L. Blackburn, G. Martin, and E. Katsavounidis, Classical Quantum Gravity 21, S1809 (2004).

[20] N. Arnaud, F. Cavalier, M. Davier, and P. Hello, Phys. Rev. D 59, 082002 (1999).

[21] T. Pradier, N. Arnaud, M.-A. Bizouard, F. Cavalier, M. Davier, and P. Hello, Phys. Rev. D 63, 042002 (2001).

[22] W. G. Anderson and R. Balasubramanian, Phys. Rev. D 60, 102001 (1999).

[23] B. Abbott et al. (LIGO Scientific Collaboration), arXiv:grqc/0704.0943.

[24] A. Di Credico (LIGO Scientific Collaboration), Classical Quantum Gravity 22, S1051 (2005).

[25] S. Hild, P. Ajith, M. Hewitson, H. Grote, and J. R. Smith, Classical Quantum Gravity 243783 (2007).

[26] T. Akutsu, M. Ando, N. Kanda, D. Tatsumi, S. Telada, S. Miyoki, M. Ohashi, and TAMA Collaboration, Classical Quantum Gravity 23, S23 (2006).

[27] C. R. Hanna (LIGO Scientific Collaboration), Classical Quantum Gravity 23, S17 (2006).

[28] K. Kötter, I. S. Heng, M. Hewitson, K. A. Strain, G. Woan, and H. Ward, Classical Quantum Gravity 20, S895 (2003).

[29] M. Hewitson and P. Ajith, Classical Quantum Gravity 22, 4903 (2005).

[30] P. Ajith, M. Hewitson, and I. S. Heng, Classical Quantum Gravity 23, S741 (2006).

[31] L. Wen and B.F. Schutz, Classical Quantum Gravity 22, S1321 (2005). 
[32] L. Cadonati, Classical Quantum Gravity 21, S1695 (2004).

[33] M. Rakhmanov and S. Klimenko, Classical Quantum Gravity 22, S1311 (2005).

[34] S. Chatterji, A. Lazzarini, L. Stein, P. Sutton, A. Searle, and M. Tinto, Phys. Rev. D 74, 082005 (2006).

[35] P. Ajith, M. Hewitson, J. R. Smith, and K. A. Strain, Classical Quantum Gravity 23, 5825 (2006).

[36] G. B. Arfken and H. J. Weber, Mathematical Methods for Physicists (Academic Press, New York, 2001), 5th ed.

[37] P. E. Johnson and D. G. Long, IEEE Trans. Signal Process. 47, 1255 (1999).

[38] R. A. Fisher, Biometrika 10, 507 (1915).

[39] J. R. Smith, P. Ajith, H. Grote, M. Hewitson, S. Hild,
H. Lück, K. A. Strain, B. Willke, J. Hough, and K. Danzmann, Classical Quantum Gravity 23, 527 (2006).

[40] J.R. Smith, Ph.D. thesis, Albert Einstein Institute and University of Hannover, 2006.

[41] L. L. Scharf, Statistical Signal Processing: Detection, Estimation, and Time Series Analysis (Addison-Wesley, Reading, MA, 1991).

[42] A. Lazzarini, S. Bose, P. Fritschel, M. McHugh, T. Regimbau, K. Reilly, J.D. Romano, J.T. Whelan, S. Whitcomb, and B.F. Whiting, Phys. Rev. D 70, 062001 (2004).

[43] M. Hewitson, H. Grote, S. Hild, H. Lück, P. Ajith, J. R. Smith, K. A. Strain, B. Willke, and G. Woan, Classical Quantum Gravity 22, 4253 (2005). 